\title{
Stathmin 1 promotes the proliferation and malignant transformation of pancreatic intraductal papillary mucinous neoplasms
}

\author{
AKIRA WATANABE, KENICHIRO ARAKI, TAKEHIKO YOKOBORI, BOLAG ALTAN, NORIHIRO ISHII, \\ MARIKO TSUKAGOSHI, NORIO KUBO, FUMIYOSHI SAITO, HIDEKI SUZUKI and HIROYUKI KUWANO \\ Department of General Surgical Science, Gunma University Graduate School of Medicine, \\ Maebashi, Gunma 371-8511, Japan
}

Received June 28, 2015; Accepted October 27, 2016

DOI: $10.3892 / \mathrm{ol} .2017 .5603$

\begin{abstract}
Pancreatic intraductal papillary mucinous neoplasms (IPMNs) are a type of pancreatic tumor, which have been identified following improvements in diagnostic imaging. However, the malignant transformation of IPMN has been difficult to diagnose clinically. To date, the mechanisms driving the progression of IPMN to cancer remain to be fully elucidated. The present study focused on Stathmin 1 (STMN1), a protein that is associated with the development of various types of cancer. The expression of STMN1 was examined immunohistochemically in tissues from cases of IPMN. The correlation between the STMN1 staining and clinical pathological factors was evaluated, and the expression of STMN1, p27 and S-phase kinase-associated protein 2 (SKP2) were compared. High expression levels of STMN1 were significantly correlated with regions of malignancy, and was associated with high expression of SKP2, low expression of nuclear p27 and a high Ki-67 index. High expression levels of STMN1 and SKP2 were significantly correlated with the transformation of IPMN to carcinoma. In addition, within the regions of carcinoma, the expression of STMN1 was weak in regions of adenoma and high in the cancerous regions. It was concluded that the high expression of STMN1 contributed to tumor proliferation and malignant transformation in the patients with IPMN. These results suggested that characterization of the expression of STMN1 may be a promising approach for predicting malignant transformation of pancreatic intraductal papillary mucinous adenoma.
\end{abstract}

Correspondence to: Dr Akira Watanabe, Department of General Surgical Science, Gunma University Graduate School of Medicine, 3-39-22 Showa-Machi, Maebashi, Gunma 371-8511, Japan E-mail: akira_watanabe@gunma-u.ac.jp

Key words: Stathmin 1, p27, S-phase kinase-associated protein 2, pancreatic intraductal papillary mucinous neoplasm, malignant transformation

\section{Introduction}

Pancreatic cancer often has a poor prognosis due to the difficulty associated with early detection. The majority of cases of pancreatic cancer are identified at an advanced stage, resulting in estimated 5 -year survival rates of $\sim 5 \%$ worldwide (1). In an effort to enhance early detection methods, the mechanisms driving pancreatic cancer carcinogenesis are currently being investigated worldwide; however, they require further elucidation. Pancreatic intraductal papillary mucinous neoplasms (IPMNs) are a type of pancreatic epithelial tumor. The incidence of IPMNs has increased due to developments in diagnostic imaging modalities, including computer tomography and magnetic resonance imaging. IPMNs can be classified into four types, intestinal, gastric foveolar, oncocytic and pancreatobiliary, based on their histology and expression of mucin proteins. IPMN progresses from benign pancreatic intraductal papillary mucinous adenoma (IPMA) to malignant pancreatic intraductal papillary mucinous carcinoma (IPMC) $(2,3)$, however, the details of this process of carcinogenesis remain to be fully elucidated. In the present study, the factors affecting the carcinogenesis of IPMN were investigated.

p27 is a well-known tumor suppressor. It was first identified as an inhibitor of cyclin-dependent kinase (CDK) complexes in transforming growth factor $\beta$-arrested cells and was classified as a member of the Cip/Kip family of CDK inhibitors (CKIs) (4). CKIs associate with a broad spectrum of cyclin-CDK complexes to negatively regulate progression through the G1 phase of the cell cycle (5). Previously, the expression of $\mathrm{p} 27$ was reported to suppress carcinogenesis in types of cystic cancer, including ovarian tumors and pancreatic mucinous cystic tumors (6). However, the role of p27 in the progression of IPMN to IPMC remains to be elucidated.

Stathmin 1 (STMN1) is an important cytoplasmic phosphoprotein, which regulates microtubule dynamics by preventing the polymerization of tubulin and through promoting microtubule destabilization. STMN1 is expressed at high levels in various types of human malignancy and is referred to as oncoprotein 18; the expression of STMN1 correlates with tumor progression and poor prognosis in several types of cancer, including breast cancer (7), prostate 
cancer (8), gastric cancer (9), hepatocellular carcinoma (10), oral squamous cell carcinoma (11), colorectal cancer (12), malignant mesothelioma (13) and urothelial carcinoma (14). These previous studies indicate that STMN1 is a fundamental cancer-associated gene, and a potential target for diagnosis and treatment. In our previous study, STMN1 contributed to poor prognosis and cancer progression in patients with extrahepatic cholangiocarcinoma (15). Additionally, there is evidence to suggest that STMN1 regulates the function of $\mathrm{p} 27$, a cytoplasmic protein shown to regulate cell migration. For example, Baldassarre et al (16) reported that STMN1 binds to p27 in the cytoplasm of cancer cells and enhances their proliferation by suppressing p27 function. In our previous study, a similar association between STMN1 and p27 was found in the cytoplasm of extrahepatic cholangiocarcinoma cells, which resulted in suppressed proliferation by inhibiting the intranuclear transport of p27 (15). The present study aimed to provide a follow up by investigating the correlation between STMN1 and p27 in the carcinogenesis of IPMN.

In the present study, the expression of STMN1 and cell cycle regulators, p27 and S-phase kinase-associated protein 2 (SKP2) were evaluated in tissue samples of IPMN using immunohistochemical analyses. The aim of the present study was to clarify the function of STMN1 in the carcinogenesis of IPMN and to investigate the mechanisms by which STMN1 drives this process of carcinogenesis.

\section{Materials and methods}

Patients and samples. The immunohistochemical analyses were performed on tissue samples obtained from 27 patients with IPMNs, who had undergone potentially curative surgery between 1995 and 2011 at Gunma University Hospital (Maebashi, Japan). All patients signed written informed consent forms as required by the institutional guidelines of Gunma University Graduate School of Medicine (Maebashi, Japan).

Immunohistochemical staining. Each $4-\mu \mathrm{m}$-thick tissue section was mounted on a silane-coated glass slide, deparaffinized, and soaked for $30 \mathrm{~min}$ at room temperature in $0.3 \%$ $\mathrm{H}_{2} \mathrm{O}_{2} /$ methanol to block endogenous peroxidases. The sections were then heated in boiling water and Immunosaver (Nishin EM, Tokyo, Japan) at $98^{\circ} \mathrm{C}$ for $45 \mathrm{~min}$. Nonspecific binding sites were then blocked by incubating the slides with Protein Block Serum-Free (Dako North America, Inc., Carpinteria, CA, USA) for $30 \mathrm{~min}$ at room temperature. Subsequently, the slides were incubated for $24 \mathrm{~h}$ at $4^{\circ} \mathrm{C}$ with the following primary antibodies (dilution, 1:100): Anti-STMN1 antibody (catalog no., sc-48362; Santa Cruz Biotechnology, Inc., Santa Cruz, CA, USA); anti-p27 antibody (catalog no., sc-56454; Santa Cruz Biotechnology, Inc.); anti-SKP2 antibody (catalog no., D3G5; Cell Signaling Technology, Inc., Danvers, MA, USA) and anti-Ki-67 antibody (catalog no., ab833; Abcam, Cambridge, UK). The primary antibody was visualized using the Histofine Simple Stain PO (Mouse) kit (Nichirei, Tokyo, Japan) with a 5-min incubation time. The 3,3'-diaminobenzidine tetrahydrochloride chromogen was applied as a $0.02 \%$ solution containing $0.005 \% \mathrm{H}_{2} \mathrm{O}_{2}$ in $50 \mathrm{mM}$ ammonium acetate-citrate acid buffer ( $\mathrm{pH}$ 6.0). The sections were lightly
Table I. Scoring criteria of immunohistochemical evaluation.

\begin{tabular}{lcccc}
\hline & \multicolumn{4}{c}{ Positively stained cells (\%) } \\
\cline { 2 - 5 } Stain intensity & 0 & $1-10$ & $10-50$ & $\geq 50$ \\
\hline 0 & Score 0 & Score 0 & Score 0 & Score 0 \\
$1+$ & Score 0 & Score 1 & Score 2 & Score 3 \\
$2+$ & Score 0 & Score 2 & Score 4 & Score 6 \\
$3+$ & Score 0 & Score 3 & Score 6 & Score 9 \\
\hline
\end{tabular}

counterstained with Mayer's hematoxylin and mounted. Negative controls were established by omitting the primary antibody. As it was previously confirmed that esophageal carcinomas expressed STMN1, p27 and SKP2 (17), esophageal carcinoma tissues that were preserved in our department were used as a positive control.

The protocol for the subsequent analysis of the immunohistochemical staining has been described in previous reports $(15,18)$. The intensity of the STMN1, p27 and SKP2 staining was scored as no expression (0), weak positive expression (1+), positive expression $(2+)$ or strong positive expression (3+). In addition, the number of tumor cells within each tissue sample were counted, and the percentage of positively stained cells was determined. All the samples were then scored as $0,1,3,4,6$ or 9, according to the criteria presented in Table I. Tissue samples with scores of 0-4 were considered negative for expression, whereas tissue samples with scores of 6-9 were considered positive for expression. Each case was evaluated by two observers. The expression of Ki-67 was determined as the percentage of cells with a high level of nuclear expression in $\sim 1,000$ cells per sample, according to a previous report (19).

Statistical analysis. The data for the continuous variables are expressed as the mean \pm standard error of the mean. The significance of differences between the values was determined using Student's $t$-test and analysis of variance. Statistical analysis of the immunohistochemical staining was performed using the $\chi^{2}$ test. $\mathrm{P}<0.05$ was considered to indicate a statistically significant difference. All statistical analyses were performed using JMP software, version 5.01 (SAS Institute, Inc., Cary, NC, USA).

\section{Results}

Immunohistochemical staining of STMN1, SKP2 and p27 in IPMN tissue samples. The expression of STMN1 was evaluated using immunohistochemistry in 27 IPMN samples. Overall, 17 IPMN samples (41.2\%) were negative for the expression of STMN1 (Fig. 1A), whereas 10 samples (58.8\%) exhibited positive cytoplasmic staining for STMN1 (Fig. 1B). Although no correlations were found between the expression of STMN1 and age, gender, cystic diameter or presence of an intracystic nodule, the samples exhibiting significantly high expression levels of STMN1 tended to be from patients diagnosed with IPMC (Table II). In the samples, high expression levels of STMN1 were also associated with high expression 
Table II. Correlation between expression levels of STMN1 and clinicopathological factors using immunohistochemistry.

\begin{tabular}{|c|c|c|c|}
\hline Factor & $\begin{array}{c}\text { STMN1 } \\
\text { low }(n=17)\end{array}$ & $\begin{array}{c}\text { STMN1 } \\
\text { high }(n=10)\end{array}$ & P-value \\
\hline Age (years) & & & 0.4007 \\
\hline$<60$ & 6 & 2 & \\
\hline$\geq 60$ & 11 & 8 & \\
\hline Gender & & & 0.2847 \\
\hline Male & 5 & 5 & \\
\hline Female & 12 & 5 & \\
\hline Cystic diameter (mm) & & & 0.9521 \\
\hline$<30$ & 10 & 6 & \\
\hline$\geq 30$ & 7 & 4 & \\
\hline Intracystic nodule & & & 0.1588 \\
\hline- & 13 & 5 & \\
\hline+ & 4 & 5 & \\
\hline Diagnosis & & & $0.0004^{\mathrm{a}}$ \\
\hline IPMA & 15 & 2 & \\
\hline IPMC & 2 & 8 & \\
\hline $\begin{array}{l}\text { Histological } \\
\text { subclassification }\end{array}$ & & & 0.262 \\
\hline Gastric & 5 & 2 & \\
\hline Intestinal & 14 & 5 & \\
\hline Pancreatobiliary & 1 & 2 & \\
\hline Oncocytic & 0 & 1 & \\
\hline SKP2 expression & & & $0.0286^{\mathrm{a}}$ \\
\hline Low & 15 & 5 & \\
\hline High & 2 & 5 & \\
\hline \multicolumn{4}{|l|}{ Nuclear p27 expression } \\
\hline Low & 7 & 8 & $0.0499^{\mathrm{a}}$ \\
\hline High & 10 & 2 & \\
\hline \multicolumn{4}{|l|}{ Ki-67 labeling index } \\
\hline (permille), mean $\pm \mathrm{SD}$ & $9.71 \pm 10.4$ & $78.8 \pm 58.8$ & $0.0002^{\mathrm{a}}$ \\
\hline
\end{tabular}

aStatistically significant $(\mathrm{P}<0.05)$. Data represent the number of patients in each category unless otherwise indicated. STMN1, Stathmin 1; IPMA, intraductal papillary mucinous adenoma; IPMC, intraductal papillary mucinous carcinoma; SKP2, S-phase kinase-associated protein 2; SD, standard deviation.

levels of SKP2, low nuclear expression levels of p27 and a high Ki-67 index (Fig. 2). No correlations were found between the expression of STMN1 and the histological subclassification of IPMN (Table I). Of note, the samples with low expression levels of STMN1 and SKP2 tended to be IPMA, whereas those with high expression levels of STMN1 and SKP2 tended to be IPMC (Table III).

Upregulation of STMN1 in cancerous areas of IPMN tissue samples. As the results of the immunohistochemical staining detected an association between high expression levels of STMN1 and IPMC, the present study evaluated the localization of STMN1 in the IPMC cases. The images in Fig. 3A-C
Table III. Correlation between STMN1 and SKP2.

\begin{tabular}{lcccc}
\hline & \multicolumn{3}{c}{ Expression of STMN1/SKP2 (n) } & \\
\cline { 2 - 4 } Diagnosis & Low/low & $\begin{array}{c}\text { High/low } \\
\text { or low/high }\end{array}$ & High/high & P-value \\
\hline IPMA & 13 & 2 & 1 & $<0.05$ \\
IPMC & 1 & 5 & 5 &
\end{tabular}

STMN1, Stathmin 1; SKP2, S-phase kinase-associated protein 2; IPMA, intraductal papillary mucinous adenoma; IPMC, intraductal papillary mucinous carcinoma.

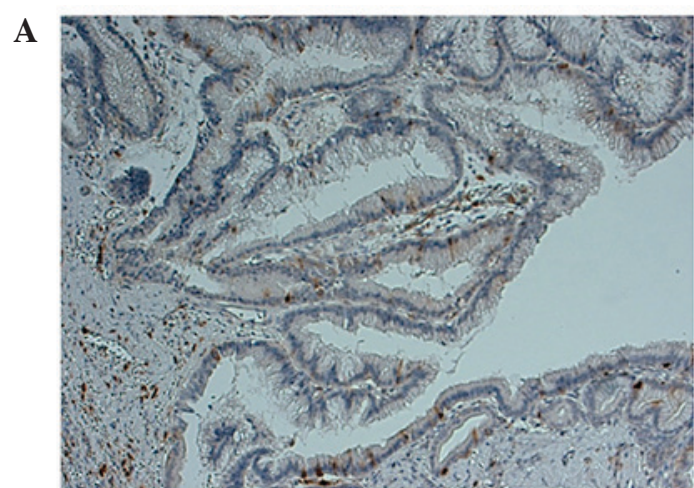

B

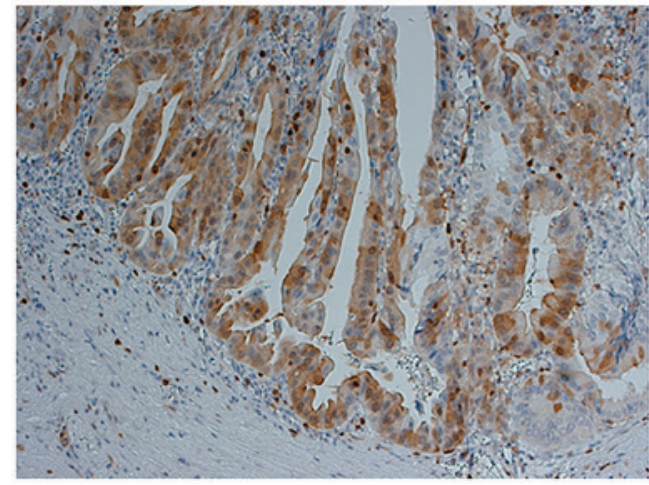

Figure 1. Immunohistochemical staining of STMN1 in primary intraductal papillary mucinous neoplasm samples. (A) Low expression of STMN1 in a primary intraductal papillary mucinous adenoma tissue specimen (magnification, x200). (B) High expression of STMN1 in a primary intraductal papillary mucinous carcinoma tissue specimen (magnification, x200). STMN1, Stathmin 1.

show the adenomatous and cancerous areas in each of three IPMC cases. In each case, the expression of STMN1 was weak in the adenomatous regions and high in the cancerous regions (Fig. 3A-C).

\section{Discussion}

In the present study, it was demonstrated that high expression levels of STMN1 were associated with tumor proliferation and malignant transformation in IPMN tissue samples. The cases of IPMC tended to have high expression levels of STMN1. The increased expression of STMN1 correlated with high expression levels of SKP2, low nuclear expression levels of p27 and a high Ki-67 index. In addition, STMN1 was expressed at high 
STMN1

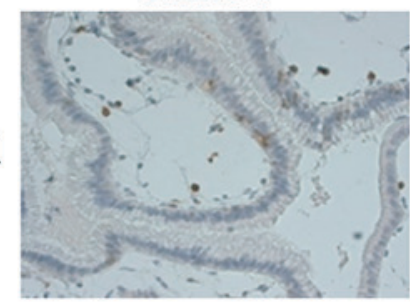

Case 1

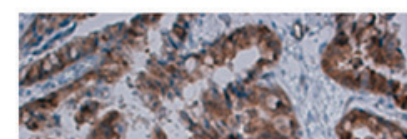

Case 2
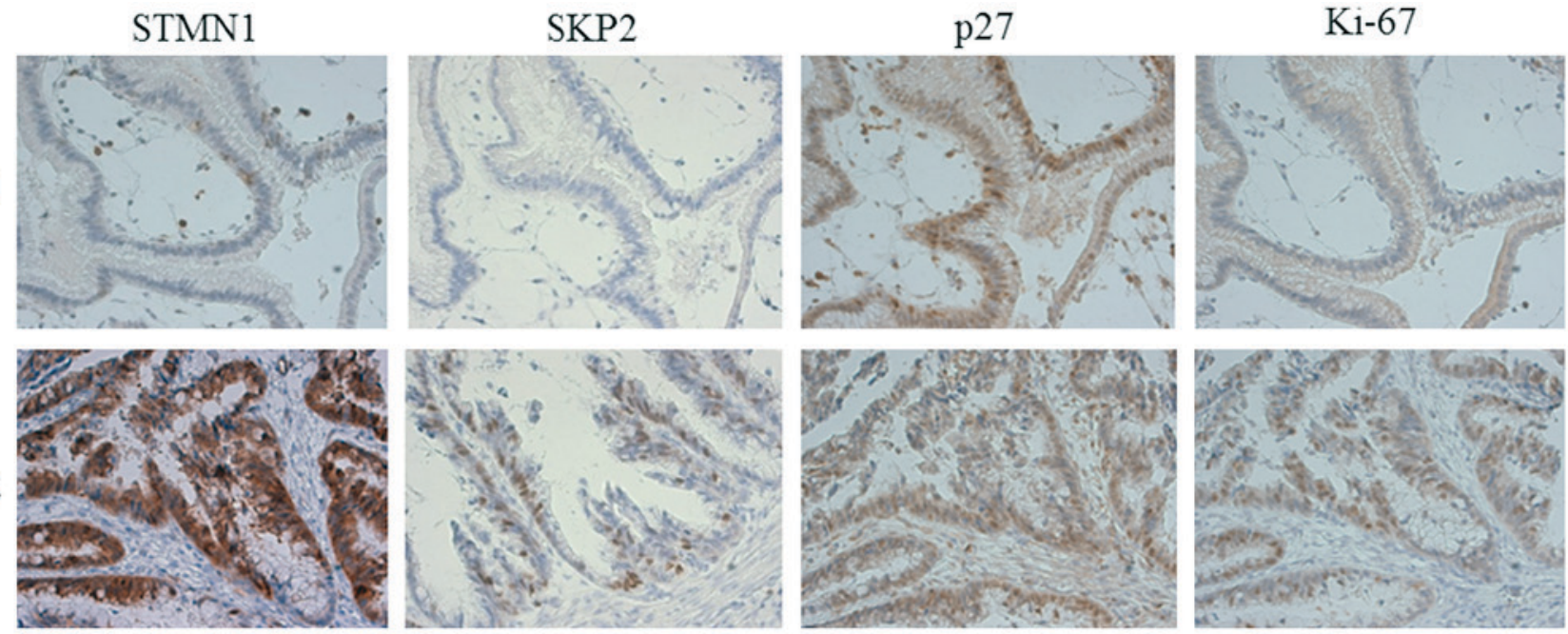

Figure 2. Immunohistochemical staining of STMN1, SKP2, p27 and Ki-67 in primary intraductal papillary mucinous neoplasm samples. Images of case 1 show low expression levels of STMN1 and SKP2, high nuclear expression of p27 and a low Ki-67 index. Images of case 2 show high expression levels of STMN1 and SKP2, low nuclear expression of p27 and a high Ki-67 index. Magnification, x50. STMN1, Stathmin 1; SKP2, S-phase kinase-associated protein 2.

A

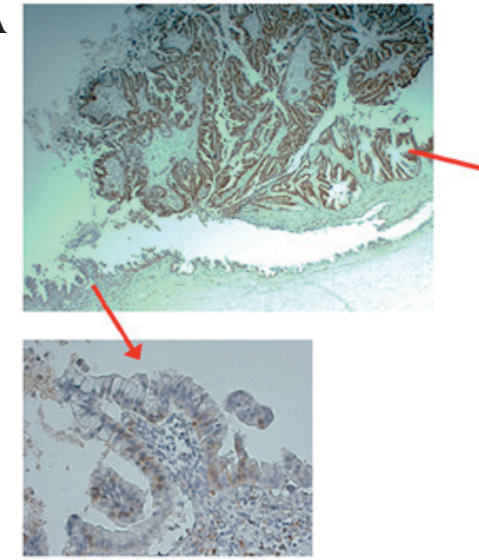

Cystic (adenomatous) area

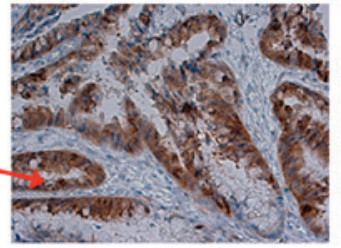

Cancerous area
B

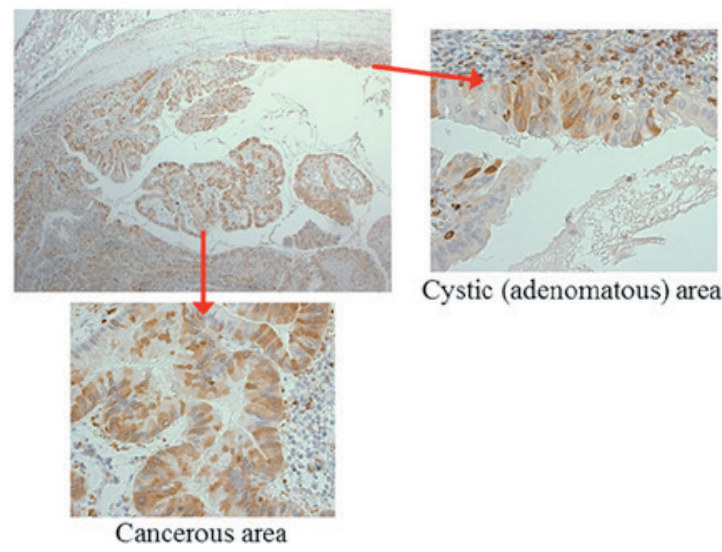

\section{C}

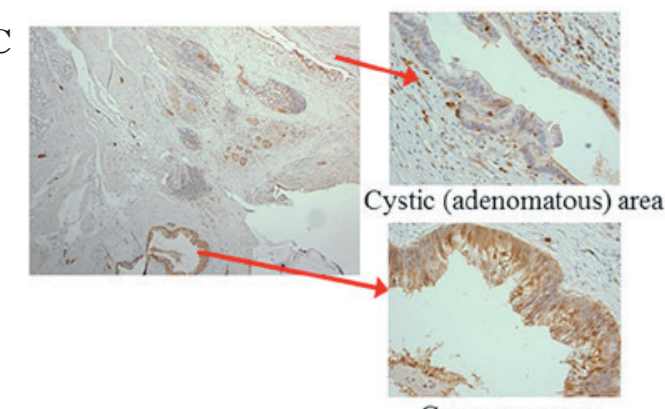

Cancerous area

Figure 3. Localization of the expression of STMN1 in primary intraductal papillary mucinous neoplasm samples using immunohistochemical staining. (A-C) Three separate cases showing tissues, in which expression levels of STMN1 were high in adenomatous areas and low in cancerous areas. Magnification, x50; magnification of insets, x200. STMN1, Stathmin 1 .

levels in the cancerous regions, compared with the adenomatous regions of the IPMC samples.

Previous reports have described an association between the expression of STMN1 and the proliferation and malignant transformation of cancer cells. Karst et al (20) reported that STMN1 is expressed at higher levels in tubal intraepithelial carcinoma and invasive carcinoma, compared with normal epithelium in pelvic serous carcinoma, and that this expression is associated with tumor initiation. Singer et al (10) reported that the expression of STMN1 is increased in hepatic carcinoma, compared with normal tissue, and that the expression of STMN1 is associated with protumorigenic events in human hepatocarcinoganesis. Additionally, Ghosh et al (8) reported the increased expression of STMN 1 in human prostate cancer, and concluded that it contributes to tumorigenesis. The results of the present study were in agreement with these previous 
reports. In analyzing the immunohistochemical staining of IPMN tissues, the cases of IPMC consistently demonstrated high expression levels of STMN1. Specifically, the expression of STMN1 was weak in adenomatous regions, but high in cancerous regions. In addition, thee tissues with high expression levels of STMN1 exhibited low nuclear expression levels of p27 and higher Ki-67 indices. These results suggested that STMN1 contributed to the proliferation and malignant transformation of IPMNs.

Previous studies have established an association between STMN1 and p27. Baldassarre et al (16) reported that STMN1 binds to p27 in the cytoplasm, and that sarcoma cells with high expression levels of STMN1 and low expression levels of p27 demonstrate increased proliferation and invasion (16). In our previous report, a similar association was found between STMN1 and p27 in extrahepatic cholangiocarcinoma, in which samples with high expression levels of STMN1 tended to have low expression levels of p27. Subsequently, STMN1 was shown to interact with p27 directly in the cytoplasm of cholangiocarcinoma cells in vitro. The knockdown of STMN1 by small interfering RNA triggered an increase in the expression of p27 and suppressed the proliferation of cholangiocarcinoma cells. It was demonstrated that STMN1 interacts with p27 in the cytoplasm, inhibiting the function of nuclear p27, thereby leading to the progression of cancer (15). In the present study, a similar inverse association was found between the expression of STMN1 and the expression of nuclear p27 in the cases of IPMN, and it was further demonstrated that these cases were associated with a high Ki-67 index, as shown in Table I. These data suggested that STMN1 inhibited the function of p27 in the nucleus, upregulating proliferation and consequently contributing to the malignant transformation of IPMNs.

SKP2 promotes the degradation of p27 via the ubiquitin-proteasome system (21). As indicated in Tables I and II, high expression levels of STMN1 were significantly correlated with high expression levels of SKP2. Additionally, low expression levels of STMN1 and SKP2 tended to be observed in IPMA, whereas high expression levels of STMN1 and SKP2 tended to be observed in the cases of IPMC. These results suggested that STMN1 and SKP2 functioned cooperatively in the malignant transformation of IPMN.

At present, it is difficult to diagnose cases of cancerous IPMN, and there is no sensitive marker to diagnose IPMC. As pancreatectomy is a high-risk surgical procedure, diagnostic markers are critically required to distinguish malignant transformation in IPMN. Bhagirath et al (22) reported that serum and urine concentrations of STMN1 were elevated in patients with bladder cancer, compared with healthy subjects, suggesting that this may be a viable marker for malignancy. In the present study, it was demonstrated that STMN1 contributed to the proliferation and malignant transformation of IPMNs. Although further investigations are required, STMN1 has the potential to be a diagnostic marker for cancer progression in IPMNs.

In conclusion, the present study demonstrated that the expression of STMN1 was correlated with tumor proliferation and malignant transformation in patients with IPMN. Therefore, evaluating the expression of STMN1 in patients with IPMN may be a useful predictor of malignant transformation. Additionally, it was demonstrated that the suppression of p27 by STMN1/SKP2 was critical for IPMN carcinogenesis. Although further investigations are required, STMN1, SKP2 and p27 may be promising therapeutic targets for IPMNs. As the present study indicated that STMN1 contributed to proliferative ability and malignant transformation in IPMN, although further investigations are required, STMN1 may offer potential as a marker for cancer progression in IPMNs.

\section{Acknowledgements}

The authors would like to thank Ms. Yukie Saito, Ms. Tomoko Yano, Ms. Tomoko Ubukata, Ms. Yuka Matsui, Ms. Ayaka Ishida and Ms. Ayaka Ishikubo (Department of General Surgical Science, Gunma University Graduate School of Medicine) for their assistance.

\section{References}

1. Poruk KE and Weiss MJ: The current state of surgery for pancreatic cancer. Minerva Gastroenterol Dietol 61: 101-115, 2015.

2. Okabayashi T, Shima Y, Kosaki T, Sumiyoshi T, Kozuki A, Iiyama T, Takezaki Y, Kobayashi M, Nishimori I, Ogawa Y and Hanazaki K: Invasive carcinoma derived from branch duct-type IPMN may be a more aggressive neoplasm than that derived from main duct-type IPMN. Oncol Lett 5: 1819-1825, 2013.

3. Nakata K, Nagai E, Ohuchida K, Aishima S, Hayashi A, Miyasaka Y, Yu J, Mizumoto K, Tanaka M and Tsuneyoshi M: REG4 is associated with carcinogenesis in the 'intestinal' pathway of intraductal papillary mucinous neoplasms. Mod Pathol 22: 460-468, 2009.

4. Nakayama K, Ishida N, Shirane M, Inomata A, Inoue T, Shishido N, Horii I, Loh DY and Nakayama K: Mice lacking p27(Kip1) display increased body size, multiple organ hyperplasia, retinal dysplasia, and pituitary tumors. Cell 85: 707-720, 1996.

5. Denicourt $C$ and Dowdy SF: Cip/Kip proteins: More than just CDKs inhibitors. Genes Dev 18: 851-855, 2004.

6. Suzuki Y, Sugiyama M, Abe N, Fujioka Y and Atomi Y: Immunohistochemical similarities between pancreatic mucinous cystic tumor and ovarian mucinous cystic tumor. Pancreas 36: e40-e46, 2008

7. Alli E, Yang JM and Hait WN: Silencing of stathmin induces tumor-suppressor function in breast cancer cell lines harboring mutant p53. Oncogene 26: 1003-1012, 2007.

8. Ghosh R, Gu G, Tillman E, Yuan J, Wang Y, Fazli L, Rennie PS and Kasper S: Increased expression and differential phosphorylation of stathmin may promote prostate cancer progression. Prostate 67: 1038-1052, 2007.

9. Jeon TY, Han ME, Lee YW, Lee YS, Kim GH, Song GA, Hur GY, Kim JY, Kim HJ, Yoon S, et al: Overexpression of stathmin1 in the diffuse type of gastric cancer and its roles in proliferation and migration of gastric cancer cells. Br J Cancer 102: 710-718, 2010.

10. Singer S, Ehemann V, Brauckhoff A, Keith M, Vreden S, Schirmacher P and Breuhahn K: Protumorigenic overexpression of stathmin/Op18 by gain-of-function mutation in p53 in human hepatocarcinogenesis. Hepatology 46: 759-768, 2007.

11. Kouzu Y, Uzawa K, Koike H, Saito K, Nakashima D, Higo M, Endo Y, Kasamatsu A, Shiiba M, Bukawa H, et al: Overexpression of stathmin in oral squamous-cell carcinoma: Correlation with tumour progression and poor prognosis. Br J Cancer 94: 717-723, 2006.

12. Zheng P, Liu YX, Chen L, Liu XH, Xiao ZQ, Zhao L, Li GQ, Zhou J, Ding YQ and Li JM: Stathmin, a new target of PRL-3 identified by proteomic methods, plays a key role in progression and metastasis of colorectal cancer. J Proteome Res 9: 4897-4905, 2010.

13. Kim JY, Harvard C, You L, Xu Z, Kuchenbecker K, Baehner R and Jablons D: Stathmin is overexpressed in malignant mesothelioma. Anticancer Res 27: 39-44, 2007.

14. Lin WC, Chen SC, Hu FC, Chueh SC, Pu YS, Yu HJ and Huang KH: Expression of stathmin in localized upper urinary tract urothelial carcinoma: Correlations with prognosis. Urology 74: 1264-1269, 2009. 
15. Watanabe A, Suzuki H, Yokobori T, Tsukagoshi M, Altan B, Kubo N, Suzuki S, Araki K, Wada S, Kashiwabara K, et al: Stathmin1 regulates p27 expression, proliferation and drug resistance, resulting in poor clinical prognosis in cholangiocarcinoma. Cancer Sci 105: 690-696, 2014

16. Baldassarre G, Belletti B, Nicoloso MS, Schiappacassi M Vecchione A, Spessotto P, Morrione A, Canzonieri V and Colombatti A: p27(Kip1)-stathmin interaction influences sarcoma cell migration and invasion. Cancer Cell 7: 51-63, 2005.

17. Akhtar J, Wang Z, Yu C, Zhang ZP and Bi MM: STMN-1 gene: A predictor of survival in stage iia esophageal squamous cell carcinoma after Ivor-Lewis esophagectomy? Ann Surg Oncol 21: 315-321, 2014

18. Altan B, Yokobori T, Mochiki E, Ohno T, Ogata K, Ogawa A, Yanai M, Kobayashi T, Luvsandagva B, Asao T and Kuwano H Nuclear karyopherin- $\alpha 2$ expression in primary lesions and metastatic lymph nodes was associated with poor prognosis and progression in gastric cancer. Carcinogenesis 34: 2314-2321, 2013.
19. Suzuki S, Miyazaki T, Tanaka N, Sakai M, Sano A, Inose T, Sohda M, Nakajima M, Kato $\mathrm{H}$ and Kuwano H: Prognostic significance of CD151 expression in esophageal squamous cell carcinoma with aggressive cell proliferation and invasiveness. Ann Surg Oncol 18: 888-893, 2011.

20. Karst AM, Levanon K, Duraisamy S, Liu JF, Hirsch MS, Hecht JL and Drapkin R: Stathmin 1, a marker of PI3K pathway activation and regulator of microtubule dynamics, is expressed in early pelvic serous carcinomas. Gynecol Oncol 123: $5-12,2011$.

21. Hao Z and Huang S: E3 ubiquitin ligase Skp2 as an attractive target in cancer therapy. Front Biosci (Landmark Ed) 20: 474-490, 2015

22. Bhagirath D, Abrol N, Khan R, Sharma M, Seth A and Sharma A: Expression of CD147, BIGH3 and Stathmin and their potential role as diagnostic marker in patients with urothelial carcinoma of the bladder. Clin Chim Acta 413: 1641-1646, 2012. 